Cinémas

Revue d'études cinématographiques

Journal of Film Studies

\title{
L'éternité au quotidien : la représentation des temps dans Les Ailes du désir de Wim Wenders
}

\section{Nicole Everaert-Desmedt}

Volume 5, numéro 1-2, automne 1994

Le Temps au cinéma

URI : https://id.erudit.org/iderudit/1001008ar

DOI : https://doi.org/10.7202/1001008ar

Aller au sommaire du numéro

Éditeur(s)

Cinémas

ISSN

1181-6945 (imprimé)

1705-6500 (numérique)

Découvrir la revue

Citer cet article

Everaert-Desmedt, N. (1994). L'éternité au quotidien : la représentation des temps dans Les Ailes du désir de Wim Wenders. Cinémas, 5(1-2), 105-122.

https://doi.org/10.7202/1001008ar
Résumé de l'article

Au cours du film Les Ailes du désir (Wim Wenders, 1987), différentes conceptions de la temporalité sont confrontées : l'éternité des anges, la vie quotidienne et mortelle des hommes, et l'intemporel vécu dans l'instant présent, une sorte d'“ éternité au quotidien ». Ce texte examine, à la lumière des catégories de Peirce, ces différentes conceptions de la temporalité, et retrace, sur le modèle du carré sémiotique de Greimas, le parcours que le récit accomplit à travers la temporalité. 


\title{
L'éternité au quotidien : la représentation des temps dans Les Ailes du désir de Wim Wenders
}

\section{Nicole Everaert-Desmedt}

\begin{abstract}
RÉSUMÉ
Au cours du film Les Ailes du désir (Wim Wenders, 1987), différentes conceptions de la temporalité sont confrontées: l'éternité des anges, la vie quotidienne et mortelle des hommes, et l'intemporel vécu dans l'instant présent, une sorte d'"éternité au quotidien". Ce texte examine, à la lumière des catégories de Peirce, ces différentes conceptions de la temporalité, et retrace, sur le modèle du carré sémiotique de Greimas, le parcours que le récit accomplit à travers la temporalité.
\end{abstract}

ABSTRACT

In the course of the film The Wings of Desire (Wim Wenders, 1987), differing conceptions of temporality confront each other: the eternity of angels, the daily life of mortal men, and the timelessness of the lived present, a sort of "eternity within daily life". Using Peirce's categories, this text looks at these different conceptions of temporality and traces, on the model of Greimas' semiotic square, the itinerary followed by the plot through temporality.

Le film de Wenders, Les Ailes du désir, est par excellence un film sur le temps. L'histoire racontée est, en effet, celle d'un passage d'une temporalité à une autre: l'ange Damiel renonce à son éternité d'ange pour naître au temps mortel. Toutefois, nous montrerons que le parcours de Damiel ne s'arrête pas là, mais 
que l'accès à la temporalité humaine est pour lui la condition à partir de laquelle il pourra atteindre une nouvelle intemporalité d'un tout autre ordre.

Nous examinerons, à la lumière des catégories de Peirce, les différentes conceptions de la temporalité qui sont confrontées au cours du film, et nous retracerons, sur le modèle du carré sémiotique de Greimas, le parcours que le récit accomplit à travers la temporalité.

Du point de vue de l'expression filmique, le récit se découpe en deux parties, la première étant essentiellement en noir et blanc, la seconde en couleurs. La première partie, qui correspond à la situation initiale du récit, est beaucoup plus longue que la seconde : elle occupe presque cinq bobines sur un total de sept. Le passage du noir et blanc à la couleur, qui se produit au plan $5058^{1}$, correspond à la transformation narrative: le passage que Damiel effectue de l'état d'ange à l'état d'homme. Mais si la transformation apparaît soudainement par l'expression colorée, nous verrons qu'elle est progressivement annoncée au cours de la première partie, lorsque Damiel éprouve un manque, et progressivement assumée au cours de la seconde partie, lorsque Damiel découvre peu à peu les joies et les limites de sa nouvelle condition. Dans la situation finale, Damiel accède, avec Marion, à la plénitude de la condition humaine. Nous étudierons donc la représentation de la temporalité tout d'abord dans la situation initiale, ensuite au cours de la transformation, et enfin dans la situation finale.

\section{La situation initiale : le temporel opposé à l'intemporel}

Pendant la première partie du film, l'intemporel s'oppose au temporel: l'éternité des anges est confrontée à la vie quotidienne et mortelle des hommes.

L'opposition entre le temporel humain et l'intemporel des anges consiste en une opposition entre la discontinuité et la continuité. La vie quotidienne des hommes est montrée par bribes ou par flashes, en discontinuité. Mais le regard des anges, exprimé par le mouvement glissant et tournoyant de la caméra, rassemble tous ces fragments de réel en une continuité filmique. 


\section{- L'INTEMPOREL : LE MONDE DES ANGES}

La continuité caractérise les anges, à tous les points de vue: leur mode de connaissance, leur présence actorielle, leur activité, leur spatialité et, bien sûr, leur temporalité.

Les anges sont omniscients: ils ont toute connaissance, "depuis toujours" et "à jamais" (p. 24). Il s'agit d'une connaissance par déduction, sans expérience sensible: "Toujours tout savoir", constate Damiel (p. 25).

Les anges, étant de purs esprits, n'ont aucune matérialité. Cela nous semble une grande réussite de la part de Wenders d'être parvenu à manifester l'immatérialité des anges malgré le corps des acteurs qui les représentent. Ces corps sont des présences absentes. Bien que circulant dans la ville, ils ne sont pas matériellement perçus par les hommes. La masse de ces corps ne fait pas écran, ils sont transparents pour les humains, ils s'effacent devant eux. Les anges sont uniformisés, rendus physiquement neutres, par leur attitude, leur costume et leur coiffure semblables.

Les anges n'agissent pas sur le plan matériel. Leur seule activité est cognitive. Elle consiste à percevoir, par la vue et l'ouïe, et à enregistrer (rassembler, conserver) ce qu'ils perçoivent pour en faire rapport (attester, certifier). Et cette activité de témoins est permanente, continue.

La perception, pour les anges, se fait à distance, uniquement par les sens de la vue et de l'ouïe. Ils ne disposent pas des autres sens, qui impliquent un contact plus matériel, comme le goût, le sens thermique, kynésique, le toucher. Leur vue saisit les formes, mais pas la couleur, trop matérielle pour eux. La couleur est en effet la plus matérielle des qualités visuelles:

C'est pourquoi, on la classe habituellement dans la théorie esthétique, du côté de la matière ou du matériau. La forme (ou la figure) peut toujours, de près ou de loin, être rapportée à une disposition intelligible et être ainsi dominée, en principe, par l'esprit. Mais la couleur, dans son être-là, paraît défier toute déduction (Lyotard, p. 163).

C'est par la vue (en noir et blanc) et l'ouïe que les anges rassemblent les fragments du réel humain. 
Ils entendent jusqu'aux pensées des hommes, qui s'expriment par des voix intérieures et qui se fondent en une continuité musicale (particulièrement dans la bibliothèque, où le mélange des voix se transforme en un choeur comme dans une cathédrale). Les voix précèdent toujours les images: on entend ce qu'entendent les anges avant de voir ce qu'ils voient. La bande sonore assure donc la continuité de la bande-image.

Comme tous les héros de Wenders, les anges sont des "professionnels du regard" (Dubois et al., p. 28). Il s'agit d'un regard en mouvement, d'un regard qui lie, qui survole et ne s'arrête pas. Le film s'ouvre par un regard: un œil apparaît sur un ciel nuageux, puis la silhouette de l'ange se détache sur la tour de l'église, regardant vers le bas, vers la ville. L'insert d'un battement d'aile suffit à indiquer que l'ange se déplace, mais c'est une caméra subjective qui prend en charge le déplacement. Pendant toute la première partie du film, la caméra est étonnamment mobile, alors que les anges sont quasi immobiles. Damiel et Cassiel apparaissent soudainement en différents lieux, mais c'est à peine s'ils y font quelques pas. Ils sont partout, sans qu'on ne les voie matériellement se déplacer, ce qui manifeste leur don d'ubiquité. Le regard des anges réunit des faits qui se passent en même temps en différents lieux. Plus particulièrement, et symboliquement, dans "le ciel au-desssus de Berlin" (titre original du film), leur regard réunit les deux parties de la ville, encore séparées par le Mur à l'époque du tournage (1987). Dans leur immatérialité, les anges ne tiennent aucun compte de la matérialité du Mur. Ils passent à travers cette frontière érigée par les hommes (plan 4013), comme à travers n'importe quel mur d'appartement. Ils se promènent à l'Est (plans 4014 à 4018) comme à l'Ouest (plan 4019). Ils traitent donc en continuité le signe par excellence de la discontinuité spatiale établie par les hommes à Berlin.

La perception des anges unifie non seulement les espaces, mais aussi les temps. Pour les anges, le temps semble fait de couches superposées qu'ils perçoivent simultanément. Cela se traduit par l'insertion de documentaires sur la ville de Berlin en 1945 , entre des vues de la ville en 1987. Lorsque les deux anges se font réciproquement leur rapport, ils commencent par rappeler ce qui se passait "il y a vingt ans aujourd'hui", "il y a cin- 
quante ans", "il y a deux cents ans", avant de relater les faits du jour. (plan 1050). Ensemble, ils se souviennent du temps où "l'Histoire n'avait pas commencé" (plan 4001), qui a duré "pendant des myriades d'années" (plan 4008). Puis ils évoquent l'histoire de l'humanité, qui se résume, de leur point de vue, en une double histoire, celle de la joie et celle des guerres.

Toutes nos observations nous amènent à conclure que, du point de vue des anges, tout est en continuité. Si l'on se tourne vers les catégories distinguées par Peirce ${ }^{2}$, on peut dire sans hésiter que les anges de Wenders relèvent pleinement de la tiercéité. La tiercéité se caractérise en effet par la continuité. La tiercéité est la catégorie de la médiation, de la synthèse, de la représentation, de la pensée, du symbolisme, de la loi :

Une loi est la manière dont un futur qui n'a pas de fin doit continuer à être (Peirce, 1.536).

[La tiercéité est une] conscience synthétique, liant entre eux les éléments du temps (Peirce, 1.377).

Les anges n'ont, par contre, aucun accès à la catégorie de la secondéité: catégorie de l'expérience, du réel, du hic et nunc, de ce qui se produit en un lieu et un temps déterminés, catégorie de la discontinuité, de la force brutale, de l'effort qui rencontre une résistance, de l'action-réaction :

[La secondéité est la] conscience d'une interruption dans le champ de la conscience, sens de la résistance, d'un fait externe, de quelque autre chose (Peirce, 1.377).

Les anges n'ont pas non plus accès à la priméité: catégorie de l'indistinction, de la qualité, du sentiment, du possible, de l'imaginaire. Pour les anges, rien n'est possible, car tout est nécessaire. Ils ne peuvent pas "deviner" (p. 25), c'est-à-dire formuler des hypothèses, faire des abductions (type de raisonnement ancré dans la priméité).

\section{- LE TEMPOREL : LE MONDE DES HOMMES}

La vie humaine s'inscrit dans la secondéité, qui se caractérise par la discontinuité, et le film met en évidence cette disconti- 
nuité sous différents aspects: actoriel (les relations des hommes entre eux), spatial et temporel.

Les relations des hommes entre eux sont régies par l'individualisme. Chaque individu s'entoure de frontières :

Chaque maître de maison, ou chaque simple propriétaire, cloue son nom sur la porte comme un blason [...] et étudie le journal comme un maitre du monde. Le peuple allemand a éclaté en autant de mini-États [...] qu'il y a d'individus [...] (Voix intérieure d'un chauffeur, p. 66).

La caméra de Wenders filme les hommes dans leur isolement. Même lorsqu'ils se trouvent ensemble dans un même espace, chacun s'isole dans ses propres pensées, et la caméra glisse de l'un à l'autre. C'est le cas, longuement, dans les moyens de transport en commun (l'avion, plans 1017 à 1025; le métro, long plan-séquence, 2003, $1 \mathrm{~min} 18 \mathrm{~s}$ ) et dans le bunker où les figurants attendent pendant le tournage du film.

Les hommes sont isolés aussi dans leurs appartements. La seule fois où deux personnes se trouvent ensemble dans une pièce, elles apparaissent dos à dos: un jeune homme devant la télévision et une femme aveugle tournée vers la fenêtre (plan 1028). Dans les appartements, l'accent est mis sur l'exiguïté de l'espace et sur la présence des murs, donc sur la discontinuité spatiale.

Le temps humain est également discontinu. La vie individuelle est limitée par la mort, qui survient brutalement, par accident. Un motocycliste blessé s'étonne de ce que ce soit "si simple que ça», de "finir ici maintenant" (p. 56). D’autres séquences insistent également sur le temps qui passe et conduit à la mort.

En attendant la mort, la vie quotidienne des hommes est remplie de choses à faire et de préoccupations d'ordre matériel ( $c f$. les voix intérieures des gens dans le métro). Les hommes se font du souci à propos de l'avenir incertain de leurs descendants ( $c f$. le grand-père et la grand-mère, p. 17-18). Il y a aussi beaucoup de temps mort dans l'existence humaine, d'ennui. Un jeune homme attend devant la télévision:

Maintenant, ça fait une heure déjà qu'on attend! Toujours rien de bien à la télé (p. 16). 
Les figurants, aussi, attendent, dans le bunker, pour le tournage du film.

L'énonciation filmique accentue la discontinuité actorielle, spatiale et temporelle en juxtaposant des situations qui introduisent divers acteurs. Ces acteurs n'ont aucun rapport entre eux, sauf celui de se trouver "par hasard" sur le passage de la caméra, autrement dit d'être perçus par les anges. Chaque acteur se trouve dans une situation externe (dans tel appartement ou telle voiture, dans le métro, sur le trottoir, dans le Lavomatic ou dans le bunker) et interne (plongé dans ses pensées, que les anges entendent), qui pourrait constituer chaque fois le point de départ d'un récit. Mais la caméra passe, sans laisser le temps à tous ces embryons de récits de "prendre " et de se développer.

Ce sont tous ces embryons de récits qui, unifiés par le regard des anges (et, conjointement, du spectateur), constituent l'histoire des hommes, une double histoire, faite de joie et de guerres (comme le constatent Damiel et Cassiel, plan 4011).

\section{La transformation : de l'intemporel au temporel \\ - UNE TRANSFORMATION BRUTALE}

La transformation se produit brutalement, au plan 5058. L'ange Damiel s'incarne, il se matérialise, il devient homme. Il passe donc de l'intemporel au temporel, de la tiercéité pure à la secondéité. La secondéité est la catégorie de l'expérience, du fait brut, de l'événement individuel qui se produit en un lieu et un temps déterminés, de la matière qui résiste. La première expérience de Damiel est précisément celle d'un choc: son armure d'ancien ange lui tombe sur la tête et le réveille.

Sur le plan de l'expression filmique, la transformation est marquée par le passage soudain à la couleur et au bruit: couleurs vives d'une fresque peinte sur le côté ouest du Mur et d'une barrière de circulation routière peinte en rouge et blanc; bruits de l'armure et d'un hélicoptère.

Juste avant la transformation, Damiel se trouve avec Cassiel du côté est du Mur. C'est là qu'il commence son processus d'incarnation: il se colore légèrement et ses traces de pas se marquent sur le sol. Devant ces indices de matérialisation imminente, Cassiel porte Damiel inanimé dans ses bras en passant à 
travers le Mur. La transformation de l'état de Damiel s'accomplit donc pendant la traversée du mur de Berlin. C'est la dernière fois qu'il peut passer à travers un obstacle matériel. Dans la deuxième partie du film, il fera l'expérience de la discontinuité humaine.

La transformation, qui a lieu brutalement, était cependant annoncée pendant la première partie du film et sera progressivement assumée par Damiel pendant la deuxième partie.

\section{- UNE TRANSFORMATION ANNONCÉE}

Dès le début du film, Damiel se montre insatisfait de n'appartenir qu’à la tiercéité :

[...] mais parfois je suis las de mon éternelle existence d'esprit (plan 1055).

$\mathrm{Au}$ cours de trois dialogues, Damiel avoue son manque et annonce son projet à Cassiel (plans 1055 à 1069, 4013 à 4019, 5046 à 5057).

Il souhaite acquérir de la matérialité, un corps qui aurait du poids et occuperait un espace délimité :

J'aimerais sentir en moi un poids qui abolisse l'illimité et m'attache à la terre (p. 23).

Sentir en marchant sa charpente qui avance (p. 25).

Si on trébuche sur mes jambes[...] on s'excusera poliment. Je me ferai bousculer et je bousculerai (p. 128)!

Il souhaite entrer dans la temporalité, vivre la discontinuité temporelle:

Pouvoir, à chaque pas, à chaque coup de vent, dire "Maintenant", et "Maintenant" et "Maintenant ", et non plus "Depuis toujours" et " $\AA$ jamais" (p. 24).

À moi-même, me conquérir une histoire (p. 88).

En avant dans l'histoire du monde (p. 89).

En avant dans le gué du temps, dans le gué de la mort (p. 128)!

Il n'envisage pas d'emblée ce qui lui semble l'enracinement le plus complet dans la temporalité humaine: 
Non que je veuille tout de suite engendrer un enfant ou planter un arbre (p. 24).

Il se contenterait du rythme de la vie quotidienne, au jour le jour:

[...] mais ce serait déjà quelque chose, au retour d'une longue journée, de nourrir le chat comme Philip Marlowe (p. 24).

Ce que Damiel souhaite atteindre en se matérialisant, donc en passant par la secondéité, c'est essentiellement la priméité. Dans son état d'ange, il souffre surtout du manque de sensations, d'exaltations sensorielles:

D'avoir la fièvre, les doigts noircis par le journal, de ne plus être exalté par l'esprit seul, mais enfin par un repas, par la courbe d'une nuque, par une oreille (p. 25).

[...] Un coup d'œil abrupt, un cri bref, une odeur âcre (p. 88).

D'abord, je prendrai un bain. Puis je me ferai raser, autant que possible par un barbier turc. Il me massera aussi jusqu'au bout des doigts (p. 128)!

Damiel récite plusieurs fois le poème sur l'enfance, qui évoque la priméité: l'indistinction, la continuité actorielle, spatiale et temporelle, la joie des sensations.

La transformation de Damiel est donc annoncée depuis le début du film, par l'aveu répété d'un manque, et aussi par l'action persuasive de deux sujets: Peter Falk et Marion. Cette transformation, annoncée sur le plan du contenu narratif, l'est également sur le plan de l'expression filmique. Pendant la partie du film en noir et blanc, l'image se colore six fois, comme pour prévenir le spectateur que la réalité humaine est plus matérielle que ce que peuvent en percevoir les anges. Le spectateur est ainsi préparé à voir l'image en couleur dès que l'ange Damiel se transforme en homme.

\section{- UNE TRANSFORMATION ASSUMÉE}

À partir du plan 5058, Damiel fait l'expérience de la matérialité, qui implique l'effort physique (son premier geste humain 
consiste à se relever), le poids des objets (il ramasse, rassemble et porte son armure sous le bras) et les obstacles (il doit s'arrêter pour laisser passer une ambulance; il se voit interdire par deux fois l'entrée du bunker où a lieu le tournage du film; c'est à travers un grillage qu'il peut serrer la main de Peter Falk; dans la salle de concert, la foule des spectateurs l'empêche de voir Marion, et il doit se faufiler parmi eux pour atteindre le bar).

Il fait l'expérience du mouvement, il doit désormais parcourir la distance entre deux espaces. Ses premiers mots d'homme sont: "En route!» Il marche d'un bon pas, il court par moments. Il a devant lui des espaces linéaires à parcourir: tout d'abord, il longe le Mur; puis on le voit de dos, parcourant une longue avenue; enfin, il avance sous la ligne du métro aérien. Quand il s'arrête pour boire son premier café, on entend dans le kiosque une chanson qui dit: "When I Go.» La rencontre entre Damiel et Marion, dans la réalité, est précédée du mouvement qu'ils font l'un et l'autre vers le lieu de leur rencontre. La caméra les suit alternativement à travers la ville, puis à l'intérieur de la salle de concert, enfin dans leur traversée du bar jusqu'au comptoir.

Damiel découvre le temps, avec grand plaisir. Il est très satisfait de sa montre, qu'il consulte fréquemment (plans 6003, 6017, 6076), et lorsque Peter Falk lui demande: "Combien de temps?", il lui répond avec enthousiasme :

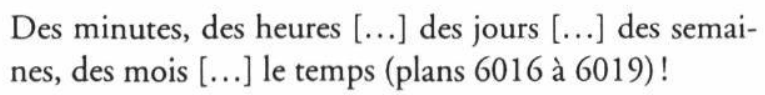

Mais Damiel se heurte aussi à l'épreuve du temps: il arrive trop tard sur le lieu du cirque pour y retrouver Marion. Il s'assied, seul, au centre de la piste, à l'endroit même où se trouvait Marion peu de temps auparavant.

Cependant une rencontre importante a lieu; Damiel parvient à attirer l'attention de Peter Falk en employant la même expression de salutation que celui-ci lui avait adressée la veille: "Compañero!" (p. 141). La communication de Damiel avec son "Destinateur ${ }^{3}$ " est très chaleureuse. Il est félicité par le "Sujet judicateur ", pour la performance qu'il a accomplie :

Je suis si heureux de te voir (p. 141)! 
Il est aussi encouragé à poursuivre sa quête par le "Sujet manipulateur" :

PETER FALK: "Qu'est-ce que tu vas faire maintenant?"

DAMIEL : "Il y a une fille[...]"

PETER FALK: “Une fille, bien!» (p. 144).

L'encouragement s'accompagne d'un rappel à l'ordre. En tant qu'ange, Damiel exprimait le désir d'avoir à "deviner enfin, au lieu de toujours tout savoir" (p. 25). Or, à présent, dans son impatience humaine, il dit à Peter Falk:

Je veux savoir! Tout savoir (p. 144)!

Aussi Damiel ne peut-il qu'acquiescer lorsque Peter Falk lui rappelle :

Tu dois trouver tout seul. C'est ce qu'il y a de beau (p. 144)!

L'autre rencontre attendue, celle de Marion, est reportée jusqu'à la situation finale...

\section{La situation finale: du temporel à l'intemporel}

La transformation a fait passer Damiel de l'intemporel au temporel, de la continuité à la discontinuité, de la tiercéité propre au monde des anges à la secondéité de la vie quotidienne humaine. Du plan 5058 au plan 7031, Damiel fait l'expérience de la matérialité, et des limites spatio-temporelles.

Mais la secondéité n'est pour Damiel que le moyen d'accès à la priméité; le temporel est pour lui un passage obligé vers un intemporel d'un autre ordre, un intemporel vécu dans l'instant présent. Une nouvelle continuité va se creuser à l'intérieur même de la discontinuité: une continuité possible, et non plus nécessaire. C'est à travers l'union charnelle, donc dans la secondéité, que Damiel et Marion pourront, ensemble, concevoir une nouvelle continuité, celle de la priméité.

L'accomplissement de la situation finale s'effectue en deux temps: le projet en est formulé dans le discours de Marion à Damiel (plans 7032 à 7035), et la confirmation en est donnée par la voix intérieure de Damiel (plans 7038 à 7049). 


\section{- LE DISCOURS DE MARION}

\section{Le contenu du discours de Marion}

Le contenu du discours de Marion s'articule sur un double rapport : "avant" est opposé à "maintenant" dans la première partie du discours, et "maintenant" est mis en relation avec "l'avenir" dans la deuxième partie.

Marion propose de marquer un arrêt dans la discontinuité qui caractérisait sa vie passée, en prenant au sérieux l'instant présent, creuset d'une nouvelle continuité.

Dans la deuxième partie du discours, Marion propose un monde comme celui dont parle Peirce pour évoquer la priméité :

Ce qu'était le monde pour Adam le jour où il ouvrit les yeux sur lui, avant qu'il n'ait établi de distinctions [...] (Peirce, 1.357).

Il s'agit d'un monde absolument nouveau :

[...] une histoire de nouveaux ancêtres (p. 167).

Il ne s'agit cependant pas de généalogie ni de nouvelle race... comme le confirmera Damiel:

Ce n'est pas un enfant mortel qui a été conçu, mais une image commune, immortelle (p. 171).

Il s'agit seulement d'un monde possible, virtuel, "invisible» :

Ce sera une histoire de géants, invisibles[...] (p. 167).

Il s'agit d'une image — donc, encore et toujours, de priméité -

Vois, mes yeux! Ils sont l'image [...] (p. 167).

Il s'agit même d'une image par excellence, d'une image invisible, qui ne sera pas montrée, pas visualisée, pas matérialisée: une priméité pure! L'image ne sera pas vue par les yeux : les yeux euxmêmes sont l'image! L'image est intérieure, et le regard est intérieur. À propos de l'importance vitale du regard intérieur, souvenons-nous de cette pensée de Marion:

À l'intérieur des yeux fermés, fermer encore les yeux $[\ldots]$ alors, même les pierres se mettent à vivre (p. 51). 


\section{L'expression filmique du discours de Marion}

La priméité pure, qui apparaît dans le contenu du discours de Marion, est évoquée également sur le plan de l'expression filmique. Le discours de Marion se présente, en effet, comme décanté des circonstances spatio-temporelles dans lesquelles il est prononcé. La décantation est obtenue par les moyens suivants:

1. Le discours est précédé d'un moment de silence, alors que Marion et Damiel se trouvent en réalité dans un lieu public, qui devrait être bondé à la fin du concert ; ensuite, une légère musique d'accompagnement remplace la musique du concert, qui était jouée réellement par un groupe connu (Nick Cave et les Bad Seeds).

2. La caméra simmobilise et fixe Marion et Damiel en plan américain, sur un fond de boiserie dorée.

3. Le seul mouvement de la caméra pendant le discours consiste à se rapprocher une fois de Marion, puis de Damiel, pour effectuer deux très gros plans de visage, qui sont les seuls de tout le film, et qui expriment l'accès à une qualité pure, à la priméité, ainsi que le note $\mathrm{G}$. Deleuze (qui se réfere lui-même à Peirce) :

Le visage réflexif exprime une Qualité pure, c'est-à-dire un "quelque chose " de commun à plusieurs objets de nature différente (p. 129).

Le gros plan n'arrache nullement son objet à un ensemble dont il ferait partie, dont il serait une partie, mais, ce qui est tout à fait différent, il l'abstrait de toutes coordonnées spatio-temporelles, c'est-à-dire il l'élève à l'état d'Entité (p. 136).

L'affect, c'est l'entité, c'est-à-dire la Puissance ou la Qualité. [...] L'image-affection, c'est la puissance ou la qualité considérées pour elles-mêmes, en tant qu'exprimées (p. 138).

Les images-affections, au sens strict, ne renvoient qu'à la priméité (p. 140).

4. À la fin du discours, lorsque Marion et Damiel s'enlacent, la caméra s'élève au-dessus d'eux et montre le bar absolument désert, bien que brillamment éclairé: un "espace quelconque", qui a perdu ses coordonnées, un espace déconnecté, vidé, devenu purement potentiel, lieu d'expression de la priméité :

L'éternité au quotidien : la représentation des temps dans Les Ailes du désir... 
Un espace quelconque n'est pas un universel abstrait, en tout temps, en tout lieu. C'est un espace parfaitement singulier, qui a seulement perdu son homogénéité, c'est-à-dire le principe de ses rapports métriques ou la connexion de ses propres parties, si bien que les raccordements peuvent se faire d'une infinité de façons. C'est un espace de conjonction virtuelle, saisi comme pur lieu du possible (Deleuze, p. 155).

5. Ensuite, l'écran est vide pendant un moment (le seul moment de tout le film), avant que n'apparaisse un plan sur la statue de l'ange de la Victoire.

\section{- LA CONFIRMATION DE DAMIEL}

\section{Le contenu de la confirmation de Damiel}

Damiel confirme tout d'abord qu'un événement a eu lieu, dans la secondéité du réel :

Il est arrivé quelque chose (p. 169).

Mais aussitôt, cet événement est décontextualisé, et la continuité s'introduit dans l'instant de cet événement :

[...] Qui continue d'arriver (p. 169).

L'indistinction caractéristique de la priméité se manifeste sur le plan temporel:

C'était vrai dans la nuit

et c'est vrai le jour (p. 169).

Il était une fois.

Il était une fois, et donc il sera (p. 171).

Lindistinction règne également sur le plan actoriel:

Qui était qui ?

J'étais en elle [...]

et elle était autour de moi (p. 169).

Je suis ensemble (p. 171).

Et sur le plan spatial. Avant leur rencontre, ni Marion ni Damiel n'habitait, en réalité, aucun lieu. Marion venait de quitter sa roulotte de cirque et Damiel, à peine fait homme, n'avait 
pas encore de pied-à-terre! Leur espace était donc totalement indéterminé, et pourtant :

Elle est venue me ramener chez moi

et j'ai trouvé ce chez-moi (p. 171).

La continuité à laquelle parviennent Marion et Damiel est de l'ordre de l'image:

Ce n'est pas un enfant mortel

qui a été conçu,

mais une image commune,

immortelle (p. 171).

Elle n'empêche pas la discontinuité humaine, réelle, celle de la mort. Mais elle aide à vivre et à mourir :

L'image que nous avons conçue

accompagnera ma mort.

J'aurai vécu dans cette image (p. 171).

Le dernier témoignage apporté par Damiel concerne le savoir. Alors que les anges sont, en principe, omniscients, Damiel peut dire et écrire :

Je sais maintenant ce qu'aucun ange ne sait (p. 172).

Il a accédé en effet au possible, dont les anges sont privés, à un mode de connaissance ancré dans la priméité, et qui trouve son fondement dans l'étonnement (première phase de toute abduction) :

Cette nuit, j'ai appris à m'étonner [...].

Ce n'est que l'étonnement

devant nous deux,

l'étonnement devant l'homme et la femme,

qui a fait de moi un être humain (p. 171).

\section{L'expression filmique de la confirmation de Damiel}

La voix intérieure de Damiel, qui parle d'image, de continuité, d'indistinction, d'étonnement, c'est-à-dire de priméité, est en contraste avec son attitude très concrète d'effort physique, caractéristique de la secondéité. Damiel est en effet occupé à

L'éternité au quotidien : la représentation des temps dans Les Ailes du désir... 
maintenir de toutes ses forces humaines la corde verticale en haut de laquelle Marion fait ses exercices, toute en souplesse et en légèreté.

Les positions sont inversées: l'ancien ange se tient en bas, au pied des escaliers, assurant un ancrage terrestre à la trapéziste qui semble presque voler autour de la corde, sous le ciel d'une verrière. L'impression céleste, de vol et de légèreté, est encore accentuée par un plan (plan 7046, 22 s) qui montre l'ombre de Marion se détachant sur un mur blanc. Il fallait que Damiel se fasse homme pour que Marion puisse se faire "ange" (notons qu'elle porte, au moment de leur rencontre dans le bar, des boucles d'oreilles en forme d'ailes) et le guider vers une nouvelle éternité. Ainsi que l'annonçait le refrain de la dernière chanson du concert de Nick Cave, entendue juste avant le discours de Marion, Damiel accédera par elle à l'éternité: "From Her to Eternity. »

\section{Synthèse : parcours du récit à travers la temporalité}

En tant qu'ange, Damiel appartient pleinement et exclusivement à la catégorie peircienne de la tiercéité, catégorie de la médiation, de la loi, donc du nécessaire et de la continuité, car une loi, dit Peirce, est «la manière dont un futur qui n'a pas de fin doit continuer à être" (Peirce, 1.536). La tiercéité est une catégorie du général et de l'intemporel. Notons $S$ la situation intemporelle dans laquelle se trouve Damiel au début du film ( $c f$. notre schéma du carré sémiotique à la prochaine page).

Cependant, dès le début du film, Damiel se montre insatisfait de n’appartenir qu’à la tiercéité, il se dit las de son "éternelle existence d'esprit" (plan 1055). Il souffre du manque de matérialisation (secondéité) et de sensations (priméité). A trois reprises, pendant la première partie du film, Damiel avoue son manque à Cassiel et lui annonce son projet de se faire homme: la situation intemporelle de départ est donc refusée; nous passons au pôle contradictoire sur le carré sémiotique: non $S$.

Le refus de l'intemporel implique le passage au temporel, c'est-à-dire à la valeur contraire sur notre carré sémiotique: $S$ '. La transformation se produit au plan 5058: Damiel est brusquement plongé dans la secondéité. Il fait l'expérience de la 
matérialité et de la discontinuité spatiale (obstacles, distances à parcourir) et temporelle (il arrive "trop tard" sur le lieu du cirque).

La secondéité n'est pas, pour Damiel, un aboutissement, mais un moyen d'accès à la priméité. La discontinuité temporelle de la mort est non pas refusée, mais dépassée par l'intensité de l'instant présent. La relation charnelle entre Marion et Damiel n'a pas abouti à la conception d'un enfant mortel: non $S$ '.

L'aboutissement du parcours de Damiel avec Marion est la conception d'une image: une image qui n'est pas montrée, pas visualisée, donc une priméité pure, de l'ordre du possible. Cette image est dite "immortelle» (p. 171). Elle permet à Damiel d'entrer dans une nouvelle continuité, à l'intérieur même de la discontinuité humaine, de vivre l'intemporel au creux de l'instant présent, l'éternité au quotidien : retour à $S$ sur notre carré sémiotique.

Nous pouvons donc représenter le parcours suivi par Damiel sur le schéma suivant :

$S$

$S^{\prime}$

INTEMPOREL

TEMPOREL

continuité

discontinuité

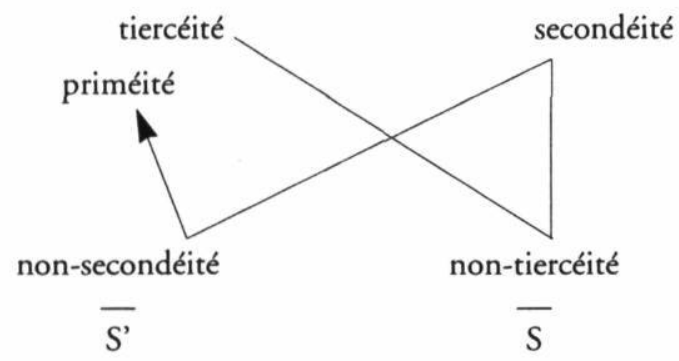

NON TEMPOREL

non-discontinuité

NON INTEMPOREL

non-continuité

Facultés universitaires Saint-Louis,

Bruxelles 


\section{NOTES}

1 Tout au long de cet article, nous renvoyons, par commodité, au texte (pages) et au découpage (plans) du film publiés dans Les Ailes du désir (Le Chesnay: Jade/ Flammarion, 1987).

2 Nous avons présenté les catégories de Peirce dans Les Processus interprétatifs. Introduction à la sémiotique de Peirce (Liège: Mardaga, 1990).

3 Rappelons que, dans la théorie actantielle, le rôle de Destinateur se décompose en "Sujet manipulateur" et "Sujet judicateur", selon les phases du déroulement narratif: le Destinateur pousse le Sujet à l'action et évalue ensuite l'action accomplie, en fonction de l'axiologie dont il est le représentant. Nous avons présenté la Sémiotique narrative dans Sémiotique du récit (Bruxelles: De Boeck-Université, 1988).

\section{OUVRAGES CITÉS}

Curot, F. "Les Ailes du désir. Complexité d'un espace filmique", Études cinématographiques, $\mathrm{n}^{\circ \mathrm{1}}$ 159-164 (1989).

Deleuze, Gilles. Cinéma 1. L'Image-mouvement. Paris: Minuit, 1983.

Dubois, P., Petit, C. et Delvaux, C. Les Voyages de Wim Wenders. Liège: Yellow Now, 1985.

Handke, Peter, Wenders, Wim. Les Ailes du désir. Le Chesnay: Jade-Flammarion, 1987.

Lyotard, J.-F. L'Inhumain. Causeries sur le temps. Paris: Galilée, 1988.

Peirce Ch. S. Collected Papers. Vol. 1-6. Cambridge/ Massachusetts: Harvard University Press, 1931-1935.

Peirce, Ch. S. Collected Papers. Vol. 7-8. Cambridge/ Massachusetts: Harvard University Press, 1958.

Schulz, M. "Les espaces du regard. Notes sur le film Der Himmel über Berlin", Espace du texte. Recueil d'hommages pour J. Geninasca (collectif). Neuchâtel: La Baconnière, 1990. 